\title{
On certain organic compounds with one mono-substitution and at least three di-substitution homogeneous derivatives
}

\author{
VaLentin Vankov ILIEV \\ Section of Algebra, Institute of Mathematics and Informatics \\ Bulgarian Academy of Sciences, 1113 Sofia, Bulgaria \\ E-mail:viliev@math.bas.bg,viliev@aubg.bg
}

\section{INTRODUCTION}

A starting point of Lunn-Senior's theory of assigning a permutation group of symmetry of degree $d$ to a given molecular structure divided into skeleton and $d$ univalent substituents is the following old observation: the number of its substitution isomers does not depend on the nature of the ligants but only on the numbers $\lambda_{k}$ of members of their different types $x_{k}, k=1,2, \ldots$, and on the skeleton. The only natural restriction is that if the skeleton contains an univalent atom (or radical), then no univalent substituent is to be identical with this atom (radical). As far as the order of ligants is irrelevant, we obtain a partition $\left(\lambda_{1}, \lambda_{2}, \ldots, \lambda_{d}\right)$ of the number $d$, that is, $\lambda_{1} \geq \lambda_{2} \geq \cdots \geq \lambda_{d} \geq 0$, and $\lambda_{1}+\lambda_{2}+\cdots+\lambda_{d}=d$. Plainly, the monomial

$$
x_{1}^{\lambda_{1}} x_{2}^{\lambda_{2}} \ldots x_{d}^{\lambda_{d}}
$$

is an exotic representation of substituents' empirical formula of the molecular structure under question. If $\Theta$ is the empirical formula of the skeleton, then

$$
\Theta x_{1}^{\lambda_{1}} x_{2}^{\lambda_{2}} \ldots x_{d}^{\lambda_{d}}
$$

is the empirical formula of the molecule. The additional information that makes difference between its empirical and structural formulae consists of a set of lists $A_{k}$, $k=1,2, \ldots, d$, each one enumerating the unsatisfied valencies of the skeleton occupied by the identical ligants of type $x_{k}$. If a numeration $1,2, \ldots, d$ of the unsatisfied valencies is fixed once and for all, then $A_{k}$ are simply pairwise disjoint subsets of the integer-valued

Research partially supported by Grant MM-1106/2001 of the Bulgarian Foundation of Scientific Research 
interval $[1, d]$, such that $[1, d]=\cup_{k} A_{k}$. Thus, the mathematical model of a structural formula of the substituents of a molecular structure with empirical formula $x_{1}^{\lambda_{1}} x_{2}^{\lambda_{2}} \ldots x_{d}^{\lambda_{d}}$, is a tabloid $A=\left(A_{1}, A_{2}, \ldots, A_{d}\right)$ with $d$ nodes of shape $\lambda=\left(\lambda_{1}, \lambda_{2}, \ldots, \lambda_{d}\right)$ :

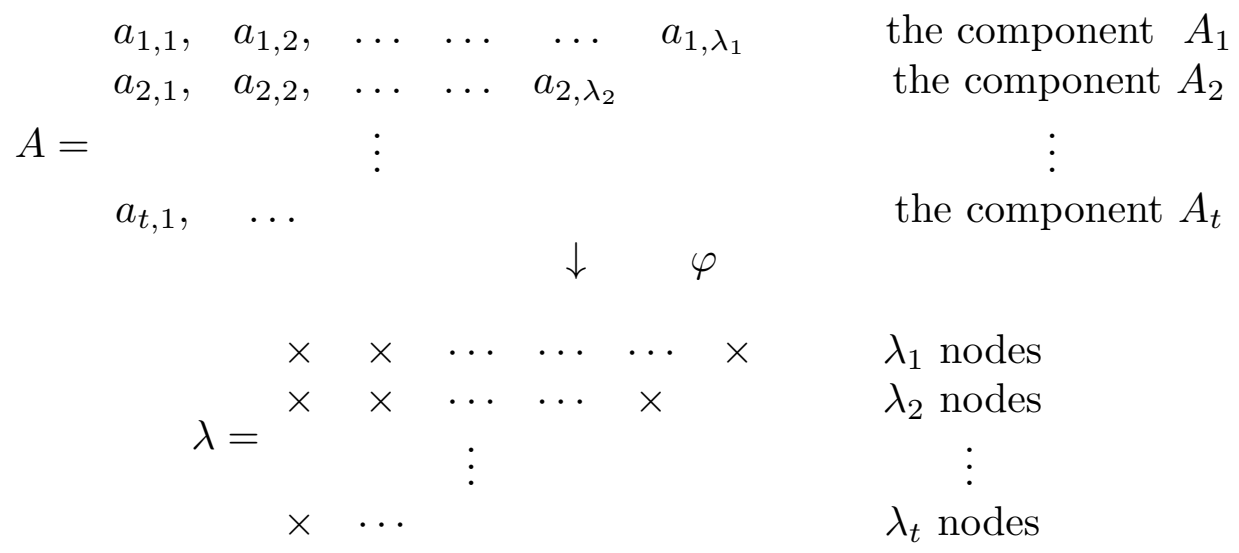

Here $\varphi: T_{d} \rightarrow P_{d}$ is the natural projection of the set $T_{d}$ of all tabloids with $d$ nodes onto the set $P_{d}$ of all partitions of $d$ that maps the tabloid $A$ onto its shape $\lambda: \lambda_{1}=\left|A_{1}\right|$, $\lambda_{2}=\left|A_{2}\right|, \ldots, \lambda_{d}=\left|A_{d}\right|$.

The structural formula of a molecule encodes its "connexity data", and does not reflect in full so called "space configuration", because the latter is a special representation of the former. "Connexity is a relation of order independent of considerations of space. The "structural" relations treated by chemists are relations of just this sort, and it is unfortunate that the word structure as used by engineers, etc., should carry with it geometrical connotations which are too special for chemistry" [6, p. 1030].

The inverse image $T_{\lambda}=\varphi^{-1}(\lambda)$ consists of all structural formulae of the substituents with empirical formula $x_{1}^{\lambda_{1}} x_{2}^{\lambda_{2}} \ldots x_{d}^{\lambda_{d}}$. The fibers $T_{\lambda}, \lambda \in P_{d}$, of the map $\varphi$ are the stages where the drama of isomerism is performed.

In [6], Lunn and Senior build in the phenomenon of isomerism of a certain type in the above mathematical model by means of action of a symmetry group $G$, consisting of permutations of the $d$ unsatisfied valencies of the skeleton, and such that any isomer of the given empirical formula $\Theta x_{1}^{\lambda_{1}} x_{2}^{\lambda_{2}} \ldots x_{d}^{\lambda_{d}}$ is represented by a $G$-orbit in $T_{\lambda}$. The group $G$ acts on the set $T_{d}$ of structural formulae by the rule

$$
\sigma\left(A_{1}, A_{2}, \ldots, A_{d}\right)=\left(\sigma\left(A_{1}\right), \sigma\left(A_{2}\right), \ldots, \sigma\left(A_{d}\right)\right)
$$

and produces the spaces $T_{\lambda ; G}$ of $G$-orbits of the structural formulae from $T_{\lambda}$. The number $n_{\lambda ; G}$ of these $G$-orbits is therefore an upper bound for the number $N_{\lambda ; \Theta}$ of experimentally known derivatives with composition $\Theta x_{1}^{\lambda_{1}} x_{2}^{\lambda_{2}} \ldots x_{d}^{\lambda_{d}}$ :

$$
N_{\lambda ; \Theta} \leq n_{\lambda ; G}
$$

for any partition $\lambda \in P_{d}$. In the cases of mono-substituted derivatives $(\lambda=(d-1,1))$, di-substituted homogeneous derivatives $(\lambda=(d-2,2))$, and di-substituted heterogeneous derivatives $\left(\lambda=\left(d-2,1^{2}\right)\right)$, the experimenters, sometimes, are certain that the corresponding numbers $N_{\lambda ; \Theta}$ attain their maximum values $n_{\lambda ; G}$. In other words, all possible $\lambda$-derivatives are prepared. In the ideal (but unattainable) situation $N_{\lambda ; \Theta}=n_{\lambda ; G}$ for all partitions $\lambda \in P_{d}$, and these equalities define the symmetry group $G$ up to so called combinatorial equivalence (See $[6, \mathrm{IV}],[7,26],[2,5.2 .5])$. 
The simple substitution reactions

$$
x_{1}^{\mu_{1}} \ldots x_{i}^{\mu_{i}} \ldots x_{j}^{\mu_{j}} \ldots \longrightarrow x_{1}^{\lambda_{1}} \ldots x_{i}^{\lambda_{i}} \ldots x_{j}^{\lambda_{j}} \ldots
$$

where $\lambda, \mu \in P_{d}$, and $\mu_{1}=\lambda_{1}, \ldots, \mu_{i}=\lambda_{i}+1, \ldots, \mu_{j}=\lambda_{j}-1, \ldots, \mu_{d}=\lambda_{d}$, that is, the replacement of a ligant of type $x_{i}$ by a ligant of type $x_{j}, j<i$, are encoded in the mathematical model via two partial orderings: on the level of empirical formulae we write $\lambda<\mu$, and on the level of the structural picture

$$
B=\left(B_{1}, B_{2}, \ldots, B_{d}\right) \longrightarrow A=\left(A_{1}, A_{2}, \ldots, A_{d}\right)
$$

$A, B \in T_{d}, \lambda=\varphi(A), \mu=\varphi(B)$, of the above simple substitution reaction, where $A$ is obtained from $B$ by moving an element $s \in B_{i}$ in the set $B_{j}$, we write $A<B$. More generally, we write $\lambda<\mu$ if $\lambda$ can be got from $\mu$ by a finite number of the above simple substitutions (this is the well known dominance order of partitions, See [5, 6.1]), and we write $A<B$ if $A$ can be obtained from $B$ via a finite sequence of the above simple movements of elements (See $[2,3.2]$. The latter ordering can be pulled down on the orbit-space $T_{d ; G}=G \backslash T_{d}: a<b$ if there are $A \in a, B \in b$ with $A<B$ (See [2, 4.1]). If $a<b, a, b \in T_{d ; G}$, the product which corresponds to $a$ can, in principle, be synthesized from the product which corresponds to $b$ via a finite sequence of simple substitution reactions. Thus, the partially ordered set $T_{d ; G}$ portrays the possible genetic relations among the derivatives of the molecule under consideration (See [2]).

In this paper we consider parent substances with molecules that can be divided into a skeleton and six univalent substituents, and have the properties mentioned in the title. Two instances are the molecules of benzene $C_{6} H_{6}$ and cyclopropane $C_{3} H_{6}$, which have one mono-substitution derivative, and three and four di-substitution homogeneous derivatives, respectively.

The paper is stratified as follows. In Section 2, Theorem 2.1 describes the Lunn-Senior's group $G$ of substitution isomerism of our compounds and Corollaries 2.7 and 2.8 give upper bounds of the numbers of their di-substitution, and tri-substitution homogeneous derivatives. In Sections 3, 4, and 5, we list the possible simple substitution reactions among di-substitution homogeneous derivatives, on one hand, and di-substitution heterogeneous, and tri-substitution homogeneous derivatives, on the other. These substitution reactions allow us to identify some derivatives with their structural formulae.

\section{The Lunn-Senior's Group of Substitution Isomerism}

The theorem below gives a characterization of the Lunn-Senior's groups of substitution isomerism of the compounds from the title.

THEOREM 2.1. If an organic compound consists of a skeleton with six univalent substituents and has one mono-substitution and at least three di-substitution homogeneous derivatives, then its Lunn-Senior's group of substitution isomerism is conjugated in $S_{6}$ either to the dihedral group

$$
\langle(123)(456),(14)(26)(35),(14)(25)(36)\rangle
$$

of order 12 , or to the cyclic group 
of order 6, or to the dihedral group

$$
\langle(123)(456),(14)(26)(35)\rangle
$$

of order 6 .

PROOF: Since there exists only one mono-substitution derivative, we have $n_{(5,1) ; G}=1$, so the Lunn-Senior's group $G \leq S_{6}$ of substitution isomerism is transitive (See [3, 3.1.1]. The existence of at least three di-substitution derivatives means that

$$
n_{(4,2) ; G} \geq 3 \text {. }
$$

Since the partition $(4,2)$ dominates the partition $\left(4,1^{2}\right)$ with respect to the dominance order, $[2,(5.3 .2)]$ implies

$$
n_{(4,2) ; G} \leq n_{\left(4,1^{2}\right) ; G} .
$$

In particular, $n_{\left(4,1^{2}\right) ; G} \geq 3$. Therefore [4, (6.1.1)] and $[4,(6.1 .2)]$ yield $g_{(4,2) ; G}=$ $g_{\left(4,1^{2}\right) ; G}=g_{(3,2,1) ; G}=g_{\left(2,1^{4}\right) ; G}=g_{\left(3,1^{3}\right) ; G}=0$. Then the linear system [3, (3.2.1)] becomes

$$
\begin{aligned}
& g_{(6) ; G}+g_{\left(3^{2}\right) ; G}+g_{\left(2^{3}\right) ; G}+g_{\left(2^{2}, 1^{2}\right) ; G}-\quad(|G|-1)=0 \\
& 2 g_{\left(3^{2}\right) ; G+}+4 g_{\left(2^{2}, 1^{2}\right) ; G}-\left(|G| n_{\left(3^{2}\right) ; G}-20\right)=0 \\
& 3 g_{\left(2^{3}\right) ; G}+3 g_{\left(2^{2}, 1^{2}\right) ; G}-\left(|G| n_{(4,2) ; G}-15\right)=0 \\
& 6 g_{\left(2^{3}\right) ; G}+6 g_{\left(2^{2}, 1^{2}\right) ; G}-\left(|G| n_{\left(2^{3}\right) ; G}-90\right)=0 \\
& 2 g_{\left(2^{2}, 1^{2}\right) ; G}-\left(|G| n_{(5,1) ; G}-6\right) \quad=0 \\
& 2 g_{\left(2^{2}, 1^{2}\right) ; G}-\left(|G| n_{\left(4,1^{2}\right) ; G}-30\right)=0 \\
& 4 g_{\left(2^{2}, 1^{2}\right) ; G}-\left(|G| n_{(3,2,1) ; G}-60\right)=0 \\
& 4 g_{\left(2^{2}, 1^{2}\right) ; G}-\left(|G| n_{\left(2^{2}, 1^{2}\right) ; G}-180\right)=0 \\
& \left(|G| n_{\left(3,1^{3}\right) ; G}-120\right)=0 \\
& \left(|G| n_{\left(2,1^{4}\right) ; G}-360\right)=0 \\
& \left(|G| n_{\left(1^{6}\right) ; G}-720\right) \quad=0
\end{aligned}
$$

Since $n_{(5,1) ; G}=1$, the fifth and sixth equalities of the system (2.4) yield $|G| \geq 6$, and

$$
|G|\left(n_{\left(4,1^{2}\right) ; G}-1\right)=24 \text {. }
$$

Then the inequality $n_{\left(4,1^{2}\right) ; G} \geq 3$ implies $|G| \leq 12$. On the other hand, the third and fourth equalities of (2.4) imply

$$
|G|\left(n_{\left(2^{3}\right) ; G}-2 n_{(4,2) ; G}\right)=60 .
$$

Thus, $|G|$ is a common divisor of 24 and 60, so we obtain two possibilities for the order $|G|$ of the group $G:|G|=12$ or $|G|=6$.

If $|G|=12$, then from (2.5) we get $n_{\left(4,1^{2}\right) ; G}=3$, and the inequalities (2.2) and (2.3) yield $n_{(4,2) ; G}=3$. Now, the fifth and the third equality of $(2.4)$ imply $g_{\left(2^{2}, 1^{2}\right) ; G}=3$ and $g_{\left(2^{3}\right) ; G}=4$. Hence the first equality of (2.4) yields $g_{(6) ; G}+g_{\left(3^{2}\right) ; G}=4$. The equality $g_{\left(3^{2}\right) ; G}=0$ is impossible since for every cycle $\sigma \in G$ of length 6 its square $\sigma^{2}$ has cyclic type $\left(3^{2}\right)$. Therefore $g_{(6) ; G}=g_{\left(3^{2}\right) ; G}=2$. Let $\sigma$ be a cycle of length 6 . After eventual conjugation, we can suppose that $\sigma^{2}=(123)(456) \in G$. Now, consider the cyclic group $K=\langle\sigma\rangle$ of order 6 and its cyclic subgroup $H=\langle(123)(456)\rangle$ that contains the two 
elements of $G$ of cyclic type $\left(3^{2}\right)$. If $\iota$ is one of the the elements of $G$ of cyclic type $\left(2^{3}\right)$, then

$$
\iota H \iota^{-1}=H,
$$

so $L=H\langle\iota\rangle$ is a subgroup of $G$ of order 6 . Now, we choose $\iota \notin K$ ( since $g_{\left(2^{3}\right) ; G}=4$ there are three elements of cyclic type $\left(2^{3}\right)$ outside $\left.K\right)$. If we suppose that $L$ is cyclic, then we would have $L=K$ (the two elements of order 6 in $G$ are in $K$ ), and in particular, $\iota \in K$ : a contradiction. Hence $L$ is isomorphic to the dihedral group of order 6 . Further, the equality (2.6) and the considerations in $[4,7.1]$ yield that we can set $\iota=(14)(26)(35)$, so $L=\langle(123)(456),(14)(26)(35)\rangle$. Now, in accord to [4, 7.3.1], we get that the group $G$ is conjugated to the group $\langle(123)(456),(14)(26)(35),(14)(25)(36)\rangle$.

If $|G|=6$, then $n_{\left(4,1^{2}\right) ; G}=5$ and the fifth equality of $(2.4)$ implies $g_{\left(2^{2}, 1^{2}\right) ; G}=0$. Then the first equality of the system $(2.4)$ becomes $g_{(6) ; G}+g_{\left(3^{2}\right) ; G}+g_{\left(2^{3}\right) ; G}=5$.

If $G$ is the cyclic group of order 6 , then it is generated, up to conjugation, by the cycle (123456), and $g_{(6) ; G}=2, g_{\left(3^{2}\right) ; G}=2$, and $g_{\left(2^{3}\right) ; G}=1$. Now, the third equality of $(2.4)$ yields $n_{(4,2) ; G}=3$.

If $G$ is the dihedral group of order 6 , then $g_{\left(3^{2}\right) ; G}=2, g_{\left(2^{3}\right) ; G}=3$, and in accordance to the third equality of $(2.4)$, we obtain $n_{(4,2) ; G}=4$. Now, we apply $[4,5.1 .1]$.

Theorem 2.1 implies immediately the following two corollaries which yield the numbers of derivatives of the molecules under consideration.

Corollary 2.7. If an organic compound consists of a skeleton with six univalent substituents and has one mono-substitution and at least three di-substitution homogeneous derivatives, and if its Lunn-Senior's group of substitution isomerism has order 12, then this compound has exactly three di-substitution homogeneous derivatives, at most three di-substitution heterogeneous derivatives, and at most three tri-substitution homogeneous derivatives.

COROLlary 2.8. If an organic compound consists of a skeleton with six univalent substituents and has one mono-substitution and at least three di-substitution homogeneous derivatives, and if its Lunn-Senior's group $G$ of substitution isomerism has order 6, then this compound has exactly three di-substitution homogeneous derivatives, at most five di-substitution heterogeneous derivatives, and at most four tri-substitution homogeneous derivatives in case $G$ is cyclic, or has three or four di-substitution homogeneous derivatives, at most five di-substitution heterogeneous derivatives, and at most four tri-substitution homogeneous derivatives in case $G$ is dihedral.

\section{Genetic Relations: the Group $G$ has Order 12}

Here we consider the possible genetic relations among the derivatives of our molecule structure in the case when its Lunn-Senior's group $G$ of substitution isomerism has order 12. An example is the benzen molecule $C_{6} H_{6}$ (See $[6, \mathrm{VI}]$ or $[1]$, or $\left.[2,6.3]\right)$. In accord to $[2,6.3]$ and Theorem 3.1, we may suppose $G=\langle(123456),(13)(46)\rangle$ and then we obtain $T_{(4,2) ; G}=\left\{a_{(4,2)}, b_{(4,2)}, c_{(4,2)}\right\}$, where:

$a_{(4,2)}$ is the $G$-orbit

$$
\{(\{1,2,4,5\},\{3,6\}),(\{2,3,5,6\},\{1,4\}),(\{1,3,4,6\},\{2,5\})\}
$$

of the tabloid $A^{(4,2)}=(\{1,2,4,5\},\{3,6\})$, 
$b_{(4,2)}$ is the $G$-orbit

$$
\begin{aligned}
& \{(\{1,2,3,4\},\{5,6\}),(\{2,3,4,5\},\{1,6\}),(\{3,4,5,6\},\{1,2\}), \\
& (\{1,4,5,6\},\{2,3\}),(\{1,2,5,6\},\{3,4\}),(\{1,2,3,6\},\{4,5\})\}
\end{aligned}
$$

of the tabloid $B^{(4,2)}=(\{1,2,3,4\},\{5,6\})$,

$c_{(4,2)}$ is the $G$-orbit

$$
\begin{aligned}
& \{(\{1,2,4,6\},\{3,5\}),(\{1,2,3,5\},\{4,6\}),(\{2,3,4,6\},\{1,5\}), \\
& (\{1,3,4,5\},\{2,6\}),(\{2,4,5,6\},\{1,3\}),(\{1,3,5,6\},\{2,4\})\}
\end{aligned}
$$

of the tabloid $C^{(4,2)}=(\{1,2,4,6\},\{3,5\})$.

Further, we get $T_{\left(3^{2}\right) ; G}=\left\{a_{\left(3^{2}\right)}, b_{\left(3^{2}\right)}, c_{\left(3^{2}\right)}\right\}$, where:

$a_{\left(3^{2}\right)}$ is the $G$-orbit

$$
\begin{gathered}
\{(\{1,2,4\},\{3,5,6\}),(\{2,3,5\},\{1,4,6\}),(\{3,4,6\},\{1,2,5\}),(\{1,4,5\},\{2,3,6\}), \\
(\{2,5,6\},\{1,3,4\}),(\{1,3,6\},\{2,4,5\}),(\{2,3,6\},\{1,4,5\}),(\{1,2,5\},\{3,4,6\}), \\
(\{1,4,6\},\{2,3,5\}),(\{3,5,6\},\{1,2,4\}),(\{2,4,5\},\{1,3,6\}),(\{1,3,4\},\{2,5,6\})\}
\end{gathered}
$$

of the tabloid $A^{\left(3^{2}\right)}=(\{1,2,4\},\{3,5,6\})$;

$b_{\left(3^{2}\right)}$ is the $G$-orbit

$$
\begin{aligned}
& \{(\{1,2,3\},\{4,5,6\}),(\{2,3,4\},\{1,5,6\}),(\{3,4,5\},\{1,2,6\}), \\
& (\{4,5,6\},\{1,2,3\}),(\{1,5,6\},\{2,3,4\}),(\{1,2,6\},\{3,4,5\})\}
\end{aligned}
$$

of the tabloid $B^{\left(3^{2}\right)}=(\{1,2,3\},\{4,5,6\})$;

$c_{\left(3^{2}\right)}$ is the $G$-orbit

$$
\{(\{1,3,5\},\{2,4,6\}),(\{2,4,6\},\{1,3,5\})\}
$$

of the tabloid $C^{\left(3^{2}\right)}=(\{1,3,5\},\{2,4,6\})$.

Moreover, we obtain

$T_{\left(4,1^{2}\right) ; G}=\left\{a_{\left(4,1^{2}\right)}, b_{\left(4,1^{2}\right)}, c_{\left(4,1^{2}\right)}\right\}$, where:

$a_{\left(4,1^{2}\right)}$ is the $G$-orbit

$$
\begin{aligned}
& \{(\{1,2,4,5\},\{3\},\{6\}),(\{2,3,5,6\},\{4\},\{1\}),(\{1,3,4,6\},\{5\},\{2\}), \\
& (\{1,2,4,5\},\{6\},\{3\}),(\{2,3,5,6\},\{1\},\{4\}),(\{1,3,4,6\},\{2\},\{5\})\}
\end{aligned}
$$

of the tabloid $A^{\left(4,1^{2}\right)}=(\{1,2,4,5\},\{3\},\{6\})$,

$b_{\left(4,1^{2}\right)}$ is the $G$-orbit

$$
\begin{gathered}
\{(\{1,2,3,4\},\{5\},\{6\}),(\{2,3,4,5\},\{6\},\{1\}),(\{3,4,5,6\},\{1\},\{2\}), \\
(\{1,4,5,6\},\{2\},\{3\}),(\{1,2,5,6\},\{3\},\{4\}),(\{1,2,3,6\},\{4\},\{5\}), \\
(\{1,2,3,4\},\{6\},\{5\}),(\{2,3,4,5\},\{1\},\{6\}),(\{3,4,5,6\},\{2\},\{1\}),
\end{gathered}
$$




$$
(\{1,4,5,6\},\{3\},\{2\}),(\{1,2,5,6\},\{4\},\{3\}),(\{1,2,3,6\},\{5\},\{4\})\}
$$

of the tabloid $B^{\left(4,1^{2}\right)}=(\{1,2,3,4\},\{5\},\{6\})$, $c_{\left(4,1^{2}\right)}$ is the $G$-orbit

$$
\begin{gathered}
\{(\{1,2,4,6\},\{3\},\{5\}),(\{1,2,3,5\},\{4\},\{6\}),(\{2,3,4,6\},\{5\},\{1\}), \\
(\{1,3,4,5\},\{6\},\{2\}),(\{2,4,5,6\},\{3\},\{1\}),(\{1,3,5,6\},\{4\},\{2\}), \\
(\{1,2,4,6\},\{5\},\{3\}),(\{1,2,3,5\},\{6\},\{4\}),(\{2,3,4,6\},\{1\},\{5\}), \\
(\{1,3,4,5\},\{2\},\{6\}),(\{2,4,5,6\},\{1\},\{3\}),(\{1,3,5,6\},\{2\},\{4\})\}
\end{gathered}
$$

of the tabloid $C^{\left(4,1^{2}\right)}=(\{1,2,4,6\},\{3\},\{5\})$.

Since

$$
\begin{gathered}
A^{\left(3^{2}\right)}<A^{(4,2)}, A^{\left(3^{2}\right)}<B^{(4,2)}, A^{\left(3^{2}\right)}<C^{(4,2)}, \\
B^{\left(3^{2}\right)}<B^{(4,2)}, B^{\left(3^{2}\right)}<(123456) C^{(4,2)}, C^{\left(3^{2}\right)}<(123456) C^{(4,2)},
\end{gathered}
$$

and since

$$
A^{\left(4,1^{2}\right)}<A^{(4,2)}, B^{\left(4,1^{2}\right)}<B^{(4,2)}, C^{\left(4,1^{2}\right)}<C^{(4,2)},
$$

we have the following inequalities

$$
\begin{aligned}
& a_{\left(3^{2}\right)}<a_{(4,2)}, a_{\left(3^{2}\right)}<b_{(4,2)}, a_{\left(3^{2}\right)}<c_{(4,2)}, \\
& b_{\left(3^{2}\right)}<b_{(4,2)}, b_{\left(3^{2}\right)}<c_{(4,2)}, c_{\left(3^{2}\right)}<c_{(4,2)},
\end{aligned}
$$

and

$$
a_{\left(4,1^{2}\right)}<a_{(4,2)}, a_{\left(4,1^{2}\right)}<b_{(4,2)}, a_{\left(4,1^{2}\right)}<c_{(4,2)} .
$$

The diagrams below represent "Körner like" relations between homogeneous di- and tri-substitution products of our molecule structure, which can be used for complete identification of these six derivatives.
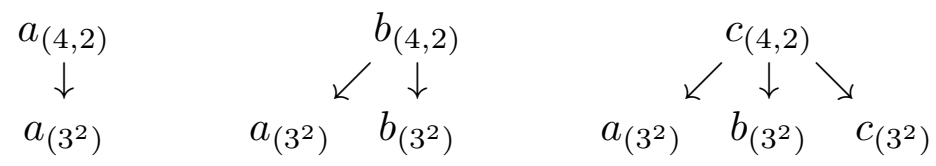

The diagrams
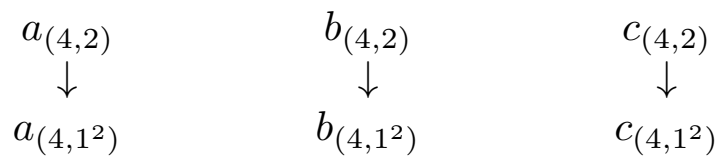

show that, as a consequence, the heterogeneous di-substitution derivatives can also be identified completely.

Here the arrow $a \rightarrow b$ means that $a>b$ and the product that corresponds to $b$ can be obtained from the product that corresponds to $a$ via a simple substitution reaction. 


\section{Genetic Relations: The Group $G$ has Order 6 and is Cyclic}

In this section we describe the genetic relations of the molecule structure under question when its Lunn-Senior's group $G$ of substitution isomerism is cyclic of order 6 . In accord with Theorem 2.1, we can suppose $G=\langle(123456)\rangle$. Then $T_{(4,2) ; G}=\left\{a_{(4,2)}, b_{(4,2)}, c_{(4,2)}\right\}$, where:

$a_{(4,2)}$ is the $G$-orbit

$$
\{(\{1,2,4,5\},\{3,6\}),(\{2,3,5,6\},\{1,4\}),(\{1,3,4,6\},\{2,5\})\},
$$

of the tabloid $A^{(4,2)}=(\{1,2,4,5\},\{3,6\})$,

$b_{(4,2)}$ is the $G$-orbit

$$
\begin{aligned}
& \{(\{1,2,3,4\},\{5,6\}),(\{2,3,4,5\},\{1,6\}),(\{3,4,5,6\},\{1,2\}), \\
& (\{1,4,5,6\},\{2,3\}),(\{1,2,5,6\},\{3,4\}),(\{1,2,3,6\},\{4,5\})\}
\end{aligned}
$$

of the tabloid $B^{(4,2)}=(\{1,2,3,4\},\{5,6\})$,

$c_{(4,2)}$ is the $G$-orbit

$$
\begin{aligned}
& \{(\{1,2,4,6\},\{3,5\}),(\{1,2,3,5\},\{4,6\}),(\{2,3,4,6\},\{1,5\}), \\
& (\{1,3,4,5\},\{2,6\}),(\{2,4,5,6\},\{1,3\}),(\{1,3,5,6\},\{2,4\})\},
\end{aligned}
$$

of the tabloid $C^{(4,2)}=(\{1,2,4,6\},\{3,5\})$.

We have $T_{\left(3^{2}\right) ; G}=\left\{a_{\left(3^{2}\right)}, b_{\left(3^{2}\right)}, c_{\left(3^{2}\right)}, d_{\left(3^{2}\right)}\right\}$, where:

$a_{\left(3^{2}\right)}$ is the $G$-orbit

$$
\begin{aligned}
& \{(\{1,2,4\},\{3,5,6\}),(\{2,3,5\},\{1,4,6\}),(\{3,4,6\},\{1,2,5\}), \\
& (\{1,4,5\},\{2,3,6\}),(\{2,5,6\},\{1,3,4\}),(\{1,3,6\},\{2,4,5\})\},
\end{aligned}
$$

of the tabloid $A^{\left(3^{2}\right)}=(\{1,2,4\},\{3,5,6\})$;

$b_{\left(3^{2}\right)}$ is the $G$-orbit

$$
\begin{gathered}
\{(\{1,2,5\},\{3,4,6\})\},(\{2,3,6\},\{1,4,5\}),(\{1,3,4\},\{2,5,6\}), \\
(\{2,4,5\},\{1,3,6\}),(\{3,5,6\},\{1,2,4\}),(\{1,4,6\},\{2,3,5\})\},
\end{gathered}
$$

of the tabloid $B^{\left(3^{2}\right)}=(\{1,2,5\},\{3,4,6\})$;

$c_{\left(3^{2}\right)}$ is the $G$-orbit

$$
\begin{aligned}
& \{(\{1,2,3\},\{4,5,6\}),(\{2,3,4\},\{1,5,6\}),(\{3,4,5\},\{1,2,6\}), \\
& (\{4,5,6\},\{1,2,3\}),(\{1,5,6\},\{2,3,4\}),(\{1,2,6\},\{3,4,5\})\}
\end{aligned}
$$

of the tabloid $C^{\left(3^{2}\right)}=(\{1,2,3\},\{4,5,6\})$;

$d_{\left(3^{2}\right)}$ is the $G$-orbit

$$
\{(\{1,3,5\},\{2,4,6\}),(\{2,4,6\},\{1,3,5\})\}
$$


of the tabloid $D^{\left(3^{2}\right)}=(\{1,3,5\},\{2,4,6\})$.

We also obtain $T_{\left(4,1^{2}\right) ; G}=\left\{a_{\left(4,1^{2}\right)}, b_{\left(4,1^{2}\right)}, c_{\left(4,1^{2}\right)}, d_{\left(4,1^{2}\right)}, e_{\left(4,1^{2}\right)}\right\}$, where: $a_{\left(4,1^{2}\right)}$ is the $G$-orbit

$$
\begin{aligned}
& \{(\{1,2,4,5\},\{3\},\{6\}),(\{2,3,5,6\},\{4\},\{1\}),(\{1,3,4,6\},\{5\},\{2\}), \\
& (\{1,2,4,5\},\{6\},\{3\}),(\{2,3,5,6\},\{1\},\{4\}),(\{1,3,4,6\},\{2\},\{5\})\}
\end{aligned}
$$

of the tabloid $A^{\left(4,1^{2}\right)}=(\{1,2,4,5\},\{3\},\{6\})$;

$b_{\left(4,1^{2}\right)}$ is the $G$-orbit

$$
\begin{aligned}
& \{(\{1,2,3,4\},\{5\},\{6\}),(\{2,3,4,5\},\{6\},\{1\}),(\{3,4,5,6\},\{1\},\{2\}), \\
& (\{1,4,5,6\},\{2\},\{3\}),(\{1,2,5,6\},\{3\},\{4\}),(\{1,2,3,6\},\{4\},\{5\})\}
\end{aligned}
$$

of the tabloid $B^{\left(4,1^{2}\right)}=(\{1,2,3,4\},\{5\},\{6\})$;

$c_{\left(4,1^{2}\right)}$ is the $G$-orbit

$$
\begin{aligned}
& \{(\{1,2,3,4\},\{6\},\{5\}),(\{2,3,4,5\},\{1\},\{6\}),(\{3,4,5,6\},\{2\},\{1\}), \\
& (\{1,4,5,6\},\{3\},\{2\}),(\{1,2,5,6\},\{4\},\{3\}),(\{1,2,3,6\},\{5\},\{4\})\}
\end{aligned}
$$

of the tabloid $C^{\left(4,1^{2}\right)}=(\{1,2,3,4\},\{6\},\{5\})$;

$d_{\left(4,1^{2}\right)}$ is the $G$-orbit

$$
\begin{aligned}
& \{(\{1,2,4,6\},\{3\},\{5\}),(\{1,2,3,5\},\{4\},\{6\}),(\{2,3,4,6\},\{5\},\{1\}), \\
& (\{1,3,4,5\},\{6\},\{2\}),(\{2,4,5,6\},\{1\},\{3\}),(\{1,3,5,6\},\{2\},\{4\})\}
\end{aligned}
$$

of the tabloid $D^{\left(4,1^{2}\right)}=(\{1,2,4,6\},\{3\},\{5\})$;

$e_{\left(4,1^{2}\right)}$ is the $G$-orbit

$$
\begin{aligned}
& \{(\{1,2,4,6\},\{5\},\{3\}),(\{1,2,3,5\},\{6\},\{4\}),(\{2,3,4,6\},\{1\},\{5\}), \\
& (\{1,3,4,5\},\{2\},\{6\}),(\{2,4,5,6\},\{3\},\{1\}),(\{1,3,5,6\},\{4\},\{2\})\}
\end{aligned}
$$

of the tabloid $E^{\left(4,1^{2}\right)}=(\{1,2,4,6\},\{5\},\{3\})$.

We have

$$
\begin{gathered}
A^{\left(3^{2}\right)}<A^{(4,2)}, A^{\left(3^{2}\right)}<B^{(4,2)}, A^{\left(3^{2}\right)}<C^{(4,2)}, \\
B^{\left(3^{2}\right)}<A^{(4,2)}, B^{\left(3^{2}\right)}<(153)(264) B^{(4,2)}, B^{\left(3^{2}\right)}<(123456) C^{(4,2)}, \\
C^{\left(3^{2}\right)<B^{(4,2)}, C^{\left(3^{2}\right)}<(123456) C^{(4,2)}, D^{\left(3^{2}\right)}<(123456) C^{(4,2)},}
\end{gathered}
$$

and

$$
\begin{gathered}
A^{\left(4,1^{2}\right)}<A^{(4,2)}, B^{\left(4,1^{2}\right)}<B^{(4,2)}, C^{\left(4,1^{2}\right)}<B^{(4,2)}, \\
D^{\left(4,1^{2}\right)}<C^{(4,2)}, E^{\left(4,1^{2}\right)}<C^{(4,2)},
\end{gathered}
$$

SO

$$
a_{\left(3^{2}\right)}<a_{(4,2)}, a_{\left(3^{2}\right)}<b_{(4,2)}, a_{\left(3^{2}\right)}<c_{(4,2)},
$$




$$
\begin{gathered}
b_{\left(3^{2}\right)}<a_{(4,2)}, b_{\left(3^{2}\right)}<b_{(4,2)}, b_{\left(3^{2}\right)}<c_{(4,2)}, \\
c_{\left(3^{2}\right)}<b_{(4,2)}, c_{\left(3^{2}\right)}<c_{(4,2)}, \\
d_{\left(3^{2}\right)}<c_{(4,2)}
\end{gathered}
$$

and

$$
\begin{gathered}
a_{\left(4,1^{2}\right)}<a_{(4,2)}, b_{\left(4,1^{2}\right)}<b_{(4,2)}, \\
c_{\left(4,1^{2}\right)}<b_{(4,2)}, d_{\left(4,1^{2}\right)}<c_{(4,2)}, e_{\left(4,1^{2}\right)}<c_{(4,2)} .
\end{gathered}
$$

The inequalities (4.1) - (4.4) indicate the existence of the corresponding (simple) substitution reactions among the $(4,2)$ - and the $\left(3^{2}\right)$-derivatives, and these substitution reactions can be used for complete identification of all $(4,2)$-derivatives. Indeed, two, three, and four $\left(3^{2}\right)$-products can be synthesized from the $(4,2)$-derivatives which correspond to $a_{(4,2)}, b_{(4,2)}$, and $c_{(4,2)}$, respectively.

The following sets of structural formulae of $\left(3^{2}\right)$-derivatives can be distinguished:

$$
\left\{a_{\left(3^{2}\right)}, b_{\left(3^{2}\right)}\right\},\left\{c_{\left(3^{2}\right)}\right\},\left\{d_{\left(3^{2}\right)}\right\} \text {. }
$$

Indeed, the products that correspond to the elements of these sets can be synthesized from three, two, and one $(4,2)$-derivatives, respectively.

The inequalities (4.5), (4.6) indicate the existence of the corresponding (simple) substitution reactions among $(4,2)$ - and $\left(4,1^{2}\right)$-derivatives, and by means of these substitution reactions we can identify the following sets of $\left(4,1^{2}\right)$-derivatives:

$$
\left\{a_{\left(4,1^{2}\right)}\right\},\left\{b_{\left(4,1^{2}\right)}, c_{\left(4,1^{2}\right)}\right\},\left\{d_{\left(4,1^{2}\right)}, e_{\left(4,1^{2}\right)}\right\} \text {. }
$$

Indeed, the product that corresponds to $a_{\left(4,1^{2}\right)}$ can be synthesized only from the identifiable $a_{(4,2)}$, the products that correspond to $b_{(4,2)}$ and $c_{\left(4,1^{2}\right)}$ can be synthesized only from the identifiable $b_{(4,2)}$, and the products that correspond to $d_{(4,2)}$ and $e_{\left(4,1^{2}\right)}$ can be synthesized only from the identifiable $c_{(4,2)}$.

\section{Genetic Relations: The Group $G$ has Order 6 and is Dihedral}

In this section we describe the genetic relations of the molecule structure under question when its Lunn-Senior's group $G$ of substitution isomerism has order 6, and is dihedral. An instance is the molecule of cyclopropane $C_{3} H_{6}$ (See [4]). In accord with Theorem 2.1, we can suppose $G=\langle(123)(456),(14)(26)(35)\rangle$. Then $T_{(4,2) ; G}=$ $\left\{a_{(4,2)}, b_{(4,2)}, c_{(4,2)}, d_{(4,2)},\right\}$, where: $a_{(4,2)}$ is the $G$-orbit

$$
\begin{aligned}
& \{(\{1,2,3,4\},\{5,6\}),(\{1,2,3,5\},\{4,6\}),(\{1,2,3,6\},\{4,5\}), \\
& (\{2,4,5,6\},\{1,3\}),(\{3,4,5,6\},\{1,2\}),(\{1,4,5,6\},\{2,3\})\}
\end{aligned}
$$

of the tabloid $A^{(4,2)}=(\{1,2,3,4\},\{5,6\})$;

$b_{(4,2)}$ is the $G$-orbit

$$
\{(\{1,2,4,5\},\{3,6\}),(\{2,3,5,6\},\{1,4\}),(\{1,3,4,6\},\{2,5\})\}
$$

of the tabloid $B^{(4,2)}=(\{1,2,4,5\},\{3,6\})$; 
$c_{(4,2)}$ is the $G$-orbit

$$
\{(\{1,2,4,6\},\{3,5\}),(\{2,3,4,5\},\{1,6\}),(\{1,3,5,6\},\{2,4\})\}
$$

of the tabloid $C^{(4,2)}=(\{1,2,4,6\},\{3,5\})$;

$d_{(4,2)}$ is the $G$-orbit

$$
\{(\{1,2,5,6\},\{3,4\}),(\{2,3,4,6\},\{1,5\}),(\{1,3,4,5\},\{2,6\})\}
$$

of the tabloid $D^{(4,2)}=(\{1,2,5,6\},\{3,4\})$.

We have

$T_{\left(3^{2}\right) ; G}=\left\{a_{\left(3^{2}\right)}, b_{\left(3^{2}\right)}, c_{\left(3^{2}\right)}, d_{\left(3^{2}\right)}\right\}$, where:

$a_{\left(3^{2}\right)}$ is the $G$-orbit

$$
\begin{aligned}
& \{(\{1,2,4\},\{3,5,6\}),(\{2,3,5\},\{1,4,6\}),(\{1,3,6\},\{2,4,5\}), \\
& (\{2,4,5\},\{1,3,6\}),(\{3,5,6\},\{1,2,4\}),(\{1,4,6\},\{2,3,5\})\},
\end{aligned}
$$

of the tabloid $A^{\left(3^{2}\right)}=(\{1,2,4\},\{3,5,6\})$;

$b_{\left(3^{2}\right)}$ is the $G$-orbit

$$
\begin{aligned}
& \{(\{1,2,5\},\{3,4,6\}),(\{2,3,6\},\{1,4,5\}),(\{1,3,4\},\{2,5,6\}), \\
& (\{1,4,5\},\{2,3,6\}),(\{2,5,6\},\{1,3,4\}),(\{3,4,6\},\{1,2,5\})\}
\end{aligned}
$$

of the tabloid $B^{\left(3^{2}\right)}=(\{1,2,5\},\{3,4,6\})$;

$c_{\left(3^{2}\right)}$ is the $G$-orbit

$$
\begin{aligned}
& \{(\{1,2,6\},\{3,4,5\}),(\{2,3,4\},\{1,5,6\}),(\{1,3,5\},\{2,4,6\}), \\
& (\{3,4,5\},\{1,2,6\}),(\{1,5,6\},\{2,3,4\}),(\{2,4,6\},\{1,3,5\})\}
\end{aligned}
$$

of the tabloid $C^{\left(3^{2}\right)}=(\{1,2,6\},\{3,4,5\})$.

$d_{\left(3^{2}\right)}$ is the $G$-orbit

$$
\{(\{1,2,3\},\{4,5,6\}),(\{4,5,6\},\{1,2,3\})\}
$$

of the tabloid $D^{\left(3^{2}\right)}=(\{1,2,3\},\{4,5,6\})$;

Moreover, we obtain

$T_{\left(4,1^{2}\right) ; G}=\left\{a_{\left(4,1^{2}\right)}, b_{\left(4,1^{2}\right)}, c_{\left(4,1^{2}\right)}, d_{\left(4,1^{2}\right)}, e_{\left(4,1^{2}\right)}\right\}$, where:

$a_{\left(4,1^{2}\right)}$ is the $G$-orbit

$$
\begin{aligned}
& \{(\{1,2,3,4\},\{5\},\{6\}),(\{1,2,3,5\},\{6\},\{4\}),(\{1,2,3,6\},\{4\},\{5\}), \\
& (\{2,4,5,6\},\{1\},\{3\}),(\{3,4,5,6\},\{2\},\{1\}),(\{1,4,5,6\},\{3\},\{2\})\}
\end{aligned}
$$

of the tabloid $A^{\left(4,1^{2}\right)}=(\{1,2,3,4\},\{5\},\{6\})$;

$b_{\left(4,1^{2}\right)}$ is the $G$-orbit

$$
\{(\{1,2,3,4\},\{6\},\{5\}),(\{1,2,3,5\},\{4\},\{6\}),(\{1,2,3,6\},\{5\},\{4\}),
$$




$$
(\{2,4,5,6\},\{3\},\{1\}),(\{3,4,5,6\},\{1\},\{2\}),(\{1,4,5,6\},\{2\},\{3\})\}
$$

of the tabloid $B^{\left(4,1^{2}\right)}=(\{1,2,3,4\},\{6\},\{5\})$;

$c_{\left(4,1^{2}\right)}$ is the $G$-orbit

$$
\begin{aligned}
& \{(\{1,2,4,5\},\{3\},\{6\}),(\{2,3,5,6\},\{1\},\{4\}),(\{1,3,4,6\},\{2\},\{5\}), \\
& (\{1,2,4,5\},\{6\},\{3\}),(\{2,3,5,6\},\{4\},\{1\}),(\{1,3,4,6\},\{5\},\{2\})\}
\end{aligned}
$$

of the tabloid $C^{\left(4,1^{2}\right)}=(\{1,2,4,5\},\{3\},\{6\})$;

$d_{\left(4,1^{2}\right)}$ is the $G$-orbit

$$
\begin{aligned}
& \{(\{1,2,4,6\},\{3\},\{5\}),(\{2,3,4,5\},\{1\},\{6\}),(\{1,3,5,6\},\{2\},\{4\}), \\
& (\{2,3,4,5\},\{6\},\{1\}),(\{1,3,5,6\},\{4\},\{2\}),(\{1,2,4,6\},\{5\},\{3\})\}
\end{aligned}
$$

of the tabloid $D^{\left(4,1^{2}\right)}=(\{1,2,4,6\},\{3\},\{5\})$;

$e_{\left(4,1^{2}\right)}$ is the $G$-orbit

$$
\begin{aligned}
& \{(\{1,2,5,6\},\{3\},\{4\}),(\{2,3,4,6\},\{1\},\{5\}),(\{1,3,4,5\},\{2\},\{6\}), \\
& (\{1,3,4,5\},\{6\},\{2\}),(\{1,2,5,6\},\{4\},\{3\}),(\{2,3,4,6\},\{5\},\{1\})\}
\end{aligned}
$$

of the tabloid $E^{\left(4,1^{2}\right)}=(\{1,2,5,6\},\{3\},\{4\})$.

This yields the inequalities

$$
\begin{aligned}
& A^{\left(3^{2}\right)}<A^{(4,2)}, A^{\left(3^{2}\right)}<B^{(4,2)}, A^{\left(3^{2}\right)}<C^{(4,2)}, \\
& B^{\left(3^{2}\right)}<(123)(456) A^{(4,2)}, B^{\left(3^{2}\right)}<B^{(4,2)}, B^{\left(3^{2}\right)}<D^{(4,2)}, \\
& C^{\left(3^{2}\right)}<(132)(465) A^{(4,2)}, C^{\left(3^{2}\right)}<C^{(4,2)}, C^{\left(3^{2}\right)}<D^{(4,2)}, \\
& D^{\left(3^{2}\right)}<A^{(4,2)},
\end{aligned}
$$

and

$$
\begin{gathered}
A^{\left(4,1^{2}\right)}<A^{(4,2)}, B^{\left(4,1^{2}\right)}<A^{(4,2)}, C^{\left(4,1^{2}\right)}<B^{(4,2)}, \\
D^{\left(4,1^{2}\right)}<C^{(4,2)}, E^{\left(4,1^{2}\right)}<D^{(4,2)},
\end{gathered}
$$

so

$$
\begin{gathered}
a_{\left(3^{2}\right)}<a_{(4,2)}, a_{\left(3^{2}\right)}<b_{(4,2)}, a_{\left(3^{2}\right)}<c_{(4,2)}, \\
b_{\left(3^{2}\right)}<a_{(4,2)}, b_{\left(3^{2}\right)}<b_{(4,2)}, b_{\left(3^{2}\right)}<d_{(4,2)}, \\
c_{\left(3^{2}\right)}<a_{(4,2)}, \quad c_{\left(3^{2}\right)}<c_{(4,2)}, c_{\left(3^{2}\right)}<d_{(4,2)}, \\
d_{\left(3^{2}\right)}<a_{(4,2)},
\end{gathered}
$$

and

$$
\begin{gathered}
a_{\left(4,1^{2}\right)}<a_{(4,2)}, b_{\left(4,1^{2}\right)}<a_{(4,2)}, \\
c_{\left(4,1^{2}\right)}<b_{(4,2)}, d_{\left(4,1^{2}\right)}<c_{(4,2)}, e_{\left(4,1^{2}\right)}<d_{(4,2)} .
\end{gathered}
$$


The inequalities (5.1) - (5.4) indicate the existence of the corresponding (simple) substitution reactions among the $(4,2)$ - and the $\left(3^{2}\right)$-derivatives, and the inequalities $(5.5)$, (5.6) indicate the existence of the corresponding (simple) substitution reactions among the $(4,2)$ - and the $\left(4,1^{2}\right)$-derivatives.

These substitution reactions can be used for distinguishing the products that correspond to different sets from the following sets of structural formulae of $(4,2)$-derivatives:

$$
\left\{a_{(4,2)}\right\},\left\{b_{(4,2)}, c_{(4,2)}, d_{(4,2)}\right\}
$$

and from the following sets of structural formulae of $\left(3^{2}\right)$-derivatives:

$$
\left\{a_{\left(3^{2}\right)}, b_{\left(3^{2}\right)}, c_{\left(3^{2}\right)}\right\},\left\{d_{\left(3^{2}\right)}\right\}
$$

Indeed, it is enough to note that from the product which corresponds to $a_{(4,2)}$ can be synthesized four $\left(3^{2}\right)$-derivatives and from the products that correspond to the elements of the set $\left\{b_{(4,2)}, c_{(4,2)}, d_{(4,2)}\right\}$, can be synthesized two $\left(3^{2}\right)$-derivatives. The products that correspond to the sets $\left\{a_{\left(3^{2}\right)}, b_{\left(3^{2}\right)}, c_{\left(3^{2}\right)}\right\}$, and $\left\{d_{\left(3^{2}\right)}\right\}$ can be synthesized from two and one $(4,2)$-derivatives, respectively.

Using the above substitution reactions, we also can identify the products corresponding to the following sets of structural formulae of $\left(4,1^{2}\right)$-derivatives:

$$
\left\{a_{\left(4,1^{2}\right)}, b_{\left(4,1^{2}\right)}\right\},\left\{c_{\left(4,1^{2}\right)}, d_{\left(4,1^{2}\right)}, e_{\left(4,1^{2}\right)}\right\} \text {. }
$$

This is because both product that correspond to $a_{\left(4,1^{2}\right)}$ and $\left.b_{\left(4,1^{2}\right)}\right\}$ can be synthesized from the identifiable $a_{(4,2)}$, and the products which correspond to $c_{\left(4,1^{2}\right)}, d_{\left(4,1^{2}\right)}$, and $e_{\left(4,1^{2}\right)}$ can be obtained from the products that correspond to $b_{(4,2)}, c_{(4,2)}$, and $d_{(4,2)}$.

\section{REFERENCES}

[1] W. Hässelbarth, The Inverse Problem of Isomer Enumeration, J. Comput. Chem. 8 (1987), $700-717$.

[2] V. V. Iliev, On Lunn-Senior's Mathematical Model of Isomerism in Organic Chemistry. Part I, MATCH - Commun. Math. Comput. Chem. 40 (1999), 153 - 186.

[3] V. V. Iliev, On the Inverse Problem of Isomer Enumeration: Part I, Case of Ethane, MATCH - Commun. Math. Comput. Chem. 43 (2001), 67 - 77.

[4] V. V. Iliev, On the Inverse Problem of Isomer Enumeration: Part II, Case of Cyclopropane, MATCH - Commun. Math. Comput. Chem. 43 (2001), 79 - 84.

[5] A. Kerber, Applied Finite Group Actions, Springer-Verlag, Berlin 1999.

[6] A. C. Lunn, J. K. Senior, Isomerism and Configuration, J. Phys. Chem. 33 (1929), $1027-1079$.

[7] G. Pólya, Kombinatorische Anzahlbestimmungen für Gruppen, Graphen und chemische Verbindungen, Acta Math. 68 (1937), 145 - 254. English translation: G. Pólya and R. C. Read, Combinatorial Enumeration of Groups, Graphs and Chemical Compounds, Springer-Verlag New York Inc., 1987. 